Andrews University

Digital Commons @ Andrews University

Honors Theses

Undergraduate Research

4-4-2019

\title{
Managing White-nose Syndrome in Bats: A Spatially Dynamic Modelling Approach
}

Mykhaylo M. Malakhov

Andrews University, mykhaylo@andrews.edu

Follow this and additional works at: https://digitalcommons.andrews.edu/honors

\section{Recommended Citation}

Malakhov, Mykhaylo M., "Managing White-nose Syndrome in Bats: A Spatially Dynamic Modelling Approach" (2019). Honors Theses. 216.

https://dx.doi.org/10.32597/honors/216

https://digitalcommons.andrews.edu/honors/216

This Honors Thesis is brought to you for free and open access by the Undergraduate Research at Digital Commons @ Andrews University. It has been accepted for inclusion in Honors Theses by an authorized administrator of Digital Commons @ Andrews University. For more information, please contact repository@andrews.edu. 


\title{
J. N. Andrews Honors Program Andrews University
}

HONS 497

Honors Thesis

Managing White-nose Syndrome in Bats: A Spatially Dynamic Modelling Approach

\author{
Mykhaylo M. Malakhov ${ }^{1}$
}

April 4, 2019

Advisor: Shandelle M. Henson

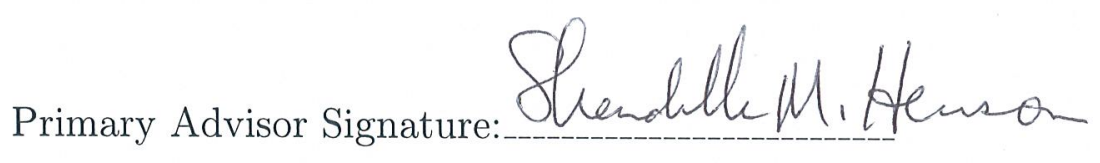

Department of Mathematics

\footnotetext{
${ }^{1}$ The following individuals will be coauthors on the version of this thesis that is submitted to a peer-reviewed journal: Jackson Barber, Julie C. Blackwood, Junyan Duan, Jordan J. Pellett, Ishan Phadke.
} 


\begin{abstract}
White-nose syndrome (WNS), caused by the invasive fungal pathogen Pseudogymnoascus destructans, is a virulent disease that has plagued North American bat populations since 2006. Over the past decade WNS has rapidly spread throughout much of the Eastern and Midwestern United States, leading to mass mortality and threatening regional extinction in a number of bat species. Thus, the need for development and implementation of effective control strategies has become increasingly exigent. While previous mathematical modelling studies have evaluated the efficacy of several proposed treatment methods, nothing is known about the impact of seasonal bat dispersal on such potential interventions. We investigate how spatial disease dynamics could affect the success of five promising WNS control strategies by posing and analysing a two-subpopulation mathematical model with migration. We demonstrate that the most effective management decisions must take interpopulation movement into account, and find that the effect of dispersal on control efficacy is mostly negative but depends on both the control combination and the primary mode of disease transmission.
\end{abstract}




\section{$1 \quad$ Introduction}

White-nose syndrome (WNS) is a rapidly spreading wildlife disease caused by the invasive fungus Pseudogymnoascus destructans ( $P$. destructans) $[1,2]$. WNS was first observed in 2006 in a cave near Albany, New York, but has since proliferated across the Eastern and Midwestern United States as well as throughout the southern provinces of Canada, and has recently been detected along the west coast of North America [3]. Wherever WNS has appeared, it has resulted in mass mortality for most hibernating bat species, many of which now face not only local, but also range-wide extinction $[4,5]$. Despite active research, WNS and its causative agent $P$. destructans remain poorly understood.

Hibernating bat species such as the little brown bat Myotis lucifugus occupy a key role in North American ecosystems, and also significantly contribute to agriculture as a form of natural pest control $[6,7]$. In light of the unprecedented population declines faced by numerous bat species, there is an imminent need to develop, test and implement control strategies for minimising WNS-related bat mortality. A variety of potential control methods targeting the determinants of WNS are currently in development; however, no tested large-scale treatments exist and the disease continues to persist unabated. Since conducting large-scale experiments on affected species is costly and potentially unethical, mathematical modelling provides a powerful way to simulate disease dynamics and thereby test proposed treatment strategies before laboratories and government agencies commit resources to trials in the wild. Multiple modelling studies have explored various aspects of WNS disease dynamics via both continuous-time and discrete-time models $[8,9,10,11,12,13,14,15]$. A few other modelling studies have considered the efficacy of several proposed control methods [16, 17].

To our knowledge no previous studies have investigated any proposed WNS control strategies in conjunction with spatial disease dynamics, despite the fact that hibernating North American bat species such as the little brown bat can perform long-distance movements [18, 19, 20]. Moreover, recent work has demonstrated that bat populations exhibit cryptic connections that bridge groups and species to pathogen dynamics, indicating that interconnectivity within and between subpopulations is an even more significant factor in WNS transmission dynamics than previously thought [21].

In this paper we investigate the effects of seasonal bat dispersal on the efficacy of five recently proposed control strategies by posing and numerically 
analysing a two-patch model with migration. We adapt our model from the one proposed in [17] by extending it to incorporate spatial dynamics, updating it to reflect recent research and considering new control strategies. In particular, we focus on the dynamics arising from the existence of dispersal between two autonomous subpopulations of bats and from the possibility of different controls in each location. We test the effectiveness of fungicide intervention, microclimate intervention, soil bacteria intervention, ultraviolet light intervention and vaccination. Our approach constitutes the first comprehensive modelling study analysing the spatial dynamics of WNS, which we examine together with control strategies targeting multiple routes of disease transmission.

\section{Methods}

\subsection{Model overview}

In this study we base our model on the biology of little brown bats. Among North American bat species affected by WNS, little brown bats face a particularly significant threat given that their populations have undergone over $90 \%$ mortality at many sites [4]. Once one of the most widely distributed bat species in the United States and Canada, the little brown bat is now predicted to become regionally extinct within the next decade $[4,11,22]$. The little brown bat is frequently used as a model organism for studying hibernating North American bat species, and has been the subject of several past modelling studies $[17,11,14]$.

Little brown bats, as well as most other North American bat species, follow three distinct annual phases: swarming, hibernation and roosting (Figure 1). Since WNS transmission dynamics remain roughly unchanged within each phase but differ drastically between phases, our model employs phase-specific equations adapted from the hybrid continuous/discrete model structure introduced in [17]. The equations in each phase track four classes of individuals: the numbers of susceptible bats $(S)$, exposed bats $(E)$, infected bats $(I)$ and $P$. destructans colony-forming units (CFUs) $(P)$.

We define susceptible bats to be those that are susceptible to $P$. destructans conidia colonisation but have not yet acquired the fungus. Some data suggest the existence of a period between $P$. destructans exposure and the manifestation of clinical WNS symptoms $[1,14]$. Thus, we also include the 
exposed class for bats that carry $P$. destructans fungus but are still asymptomatic. We assume exposed bats are not infectious and thus do not shed $P$. destructans conidia into the environment or transmit WNS via bat-tobat contact. Infected bats are both symptomatic and infectious; they die at an increased rate, shed $P$. destructans spores into the environment and transmit WNS to other bats via physical contact. Thus, the total size of bat population $i$ is $N_{i}=S_{i}+E_{i}+I_{i}$. Since $P$. destructans can grow in cold cave and mine soils as well as on bats, we include a $P_{i}$ class to account for the free-living pathogen within each population's hibernaculum.

In order to most accurately establish the effects of seasonal bat migration on population survival, we compared model dynamics arising in a spatial setting with baseline dynamics in a single population setting. That is, we first evaluated each control method on its own within a single population without migration and then extended our single-population model to incorporate two subpopulations, which we denote $A$ and $B$. When considering the model with dispersal, the two subpopulations are simulated autonomously. That is, each subpopulation occupies its own hibernaculum and thus has its own $S$, $E, I$ and $P$ classes, and the two subpopulations are not necessarily subjected to the same controls. To account for dispersal between the subpopulations, our model moves a proportion of the bats in each subpopulation to their respective class in the other subpopulation after the end of swarming (see Figure 1).

\subsection{Seasonal stages}

\subsubsection{Swarming phase}

The swarming phase lasts approximately 2 months from mid-August to midOctober (simulation days 1 through 61). During this time each subpopulation congregates near a hibernaculum to mate and accumulate fat stores in preparation for hibernation [19]. Studies suggest that the swarming phase does not play a significant role in the transmission and spread of WNS; despite high bat-to-bat contact, normal bat immune system function and high body temperatures preclude infection at this time [23]. Hence, we assume that during swarming exposed bats remain in the exposed class and do not become

infected. Since no infectious bats are present, susceptible bats can become exposed to $P$. destructans from the environment but not from bat-to-bat contact. We assume $P$. destructans grows logistically in every phase. 
The swarming phase equations are given by:

$$
\begin{aligned}
& \frac{\mathrm{d} S}{\mathrm{~d} t}=-\phi P S-\mu S \\
& \frac{\mathrm{d} E}{\mathrm{~d} t}=\phi P S-\mu E \\
& \frac{\mathrm{d} P}{\mathrm{~d} t}=\eta P\left(1-\frac{P}{K_{P d}}\right)
\end{aligned}
$$

During the swarming phase the susceptible class $(S)$ decreases at a combination of two rates: a density-dependent rate $\phi$ of disease transmission from environment-to-bat contact and the natural rate of bat mortality $\mu$. The exposed class $(E)$ increases at the density-dependent rate $\phi$ of disease transmission from environment-to-bat contact, and decreases at the natural rate of bat mortality $\mu$. The environmental $P$. destructans reservoir $(P)$ grows logistically at the natural free-living $P$. destructans growth rate $\eta$ with carrying capacity $K_{P d}$. Note that since all infected bats become exposed, susceptible or dead before the beginning of the swarming phase, the $I$ class is absent. (See Sections 2.2.4 and 2.2.5 for an explanation of the dynamics that precede the swarming phase.)

\subsubsection{Dispersal reclassification}

It is known that little brown bats, as well as other North American bat species, can perform long-distance movements throughout the swarming and roosting phases [20, 18]. Most migratory movements occur at the end of swarming, when bats can travel a considerable distance and end up hibernating in hibernacula other than those in which they swarmed. Moreover, since disease transmission occurs only during the hibernation phase, multiple migration events during the roosting and swarming phases would have no direct bearing on the force of infection. Therefore, we account for all possible between-subpopulation movements throughout the year via a single annual dispersal event occurring after the end of swarming but before the beginning of hibernation. This is a single step occurring instantaneously at which a proportion $\sigma$ of each population class $(S, E$ and $I)$ moves to the respective class in the other subpopulation.

The dispersal reclassification equation is given by:

$$
\left(\begin{array}{lll}
S_{A_{1}} & E_{A_{1}} & I_{A_{1}} \\
S_{B_{1}} & E_{B_{1}} & I_{B_{1}}
\end{array}\right)=\left(\begin{array}{cc}
1-\sigma & \sigma \\
\sigma & 1-\sigma
\end{array}\right)\left(\begin{array}{lll}
S_{A_{0}} & E_{A_{0}} & I_{A_{0}} \\
S_{B_{0}} & E_{B_{0}} & I_{B_{0}}
\end{array}\right)
$$


where $S_{A_{0}}, E_{A_{0}}, I_{A_{0}}$ and $S_{B_{0}}, E_{B_{0}}, I_{B_{0}}$ denote the susceptible, exposed and infected classes of subpopulations $A$ and $B$, respectively, at the end of the swarming phase. Likewise, $S_{A_{1}}, E_{A_{1}}, I_{A_{1}}$ and $S_{B_{1}}, E_{B_{1}}, I_{B_{1}}$ denote the resulting initial conditions for the hibernation phase. Note that the total population size does not change at the dispersal reclassification.

\subsubsection{Hibernation phase}

The hibernation phase lasts approximately 7 months from mid-October to mid-May (simulation days 62 through 273). During hibernation bats lower their basal metabolic rates and remain torpid, causing their body temperatures to drop to a mere $2-8{ }^{\circ} \mathrm{C}[24,25]$. Due to their lowered immune system function and body temperatures, bats are most susceptible to $P$. destructans infection at this time [26]. We assume that during hibernation susceptible bats can become exposed to $P$. destructans from contact with infected bats as well as with the environment. After an average of 83 days [27] exposed bats move into the infected class and become symptomatic and infectious. $P$. destructans continues to grow logistically, but during hibernation the rate is enhanced by shedding from infected bats.

The hibernation phase equations are given by:

$$
\begin{aligned}
\frac{\mathrm{d} S}{\mathrm{~d} t} & =-(\beta I+\phi P) S-\mu S \\
\frac{\mathrm{d} E}{\mathrm{~d} t} & =(\beta I+\phi P) S-(\tau+\mu) E \\
\frac{\mathrm{d} I}{\mathrm{~d} t} & =\tau E-(\delta+\mu) I \\
\frac{\mathrm{d} P}{\mathrm{~d} t} & =(\omega I+\eta P)\left(1-\frac{P}{K_{P d}}\right)
\end{aligned}
$$

All of the equation terms present in the swarming phase also exist here, with an addition of the following: The rate at which the susceptible class $(S)$ decreases is intensified by a density-dependent rate $\beta$ of transmissive bat-to-bat contact, and consequently the rate at which the exposed class $(E)$ increases is intensified by the same amount. Furthermore, the exposed class also decreases at an additional rate $\tau$ representing disease progression. Here the infected class $(I)$ appears, made up of those individuals whose disease progressed from the exposed stage to the symptomatic-infectious stage. The infected class decreases at the sum of the natural bat mortality rate $\mu$ and 
the WNS-induced mortality rate $\delta$. Finally, the free-living $P$. destructans class $(P)$ increases at an intensified rate comprised of the sum of its natural growth rate $\eta$ and the rate $\omega$ of $P$. destructans spore shedding from infected bats.

\subsubsection{Roosting reclassification}

In addition to damaging tissue and creating distinctive white lesions on the muzzles and wings of infected bats, $P$. destructans causes dehydration that leads to abnormally frequent and long arousals from torpor [28]. Due to these arousals, infected bats may use up their fat storage in winter and consequently fail to successfully transition to roosting [29]. After the end of hibernation but before the start of roosting, our model moves a proportion of infected bats to the exposed class, while the remaining infected bats die. This annual reclassification event accounts for all infected bats that do not survive into the roosting phase.

The roosting reclassification equations are given by:

$$
\begin{aligned}
& S_{1}=S_{0} \\
& E_{1}=E_{0}+\frac{1}{s \delta+1} I_{0} \\
& I_{1}=0 \\
& P_{1}=P_{0}
\end{aligned}
$$

At a single step occurring instantaneously after the end of hibernation but before the beginning of roosting, a proportion $\frac{1}{s \delta+1}$ of infected bats $(I)$ are assumed to be non-moribund and move to the exposed class $(E)$, while the remaining infected bats die. Note that the proportion depends on the rate of disease-induced mortality $\delta$ and a scaling constant $s$ chosen as in [17]. The remaining classes are not affected by the reclassification.

\subsubsection{Roosting phase}

The roosting phase lasts approximately 3 months from mid-May to midAugust (simulation days 274 through 309 and again days 331 through 365). After bats leave hibernacula in mid-May they disperse in small groups to summer roosting sites formed in trees, abandoned buildings and under piles of wood or rock, where they remain until the next swarming phase [19]. At 
this time bats have full immune system function and high body temperatures of over $20^{\circ} \mathrm{C}$, which prevents new $P$. destructans infections and allows a small number of bats to gradually clear the fungus [30,31] (thus moving from the exposed class to the susceptible class). Note that due to constant year-round conditions in hibernacula, $P$. destructans continues to grow on cave sediments despite the absence of bats [11].

The roosting phase equations are given by:

$$
\begin{aligned}
& \frac{\mathrm{d} S}{\mathrm{~d} t}=a E-\mu S \\
& \frac{\mathrm{d} E}{\mathrm{~d} t}=-a E-\mu E \\
& \frac{\mathrm{d} P}{\mathrm{~d} t}=\eta P\left(1-\frac{P}{K_{P d}}\right)
\end{aligned}
$$

The susceptible class $(S)$ increases at a rate $a$ as some exposed bats $(E)$ slowly clear their infection, and it decreases at the natural bat mortality rate $\mu$. The exposed class decreases at the same rate $a$, as well as at the natural bat mortality rate $\mu$. Since the hibernaculum environment in which freeliving $P$. destructans grows remains the same year-round [11], the equation for the $P$. destructans class $(P)$ is the same as during the swarming phase.

\subsubsection{Roosting with birth}

Little brown bats reproduce during a three-week period in the middle of the roosting phase (simulation days 310 through 330). The dynamics here are identical to those for the roosting phase with the addition of a birth term.

The birth subphase equations are given by:

$$
\begin{aligned}
\frac{\mathrm{d} S}{\mathrm{~d} t} & =\gamma N\left(1-\frac{N}{K_{M l}}\right)+a E-\mu S \\
\frac{\mathrm{d} E}{\mathrm{~d} t} & =-a E-\mu E \\
\frac{\mathrm{d} P}{\mathrm{~d} t} & =\eta P\left(1-\frac{P}{K_{P d}}\right)
\end{aligned}
$$

where $N=S+E$.

The birth subphase equations are identical to those of the roosting phase, except for the addition of a logistic growth term in the equation for the susceptible class $(S)$, which causes it to increase at an additional rate $\gamma$ with carrying capacity $K_{M l}$. 


\subsection{Control strategies}

We investigated five promising WNS treatment methods proposed in recent laboratory studies. We assumed that each subpopulation can either have one control strategy implemented or none implemented at all. Thus, in simulations of the two-subpopulation system with migration we tested all 36 possible combinations of control strategies, including combinations where both subpopulations have the same control strategy and combinations where the implemented control strategies are different between subpopulations. While strategies can differ between subpopulations, both strategies are implemented at the same intervention intensity $\alpha \in[0,1]$.

\subsubsection{Fungicide intervention}

We considered fungicide intervention in the form of a chemical treatment that persists in the hibernaculum throughout the year, limiting the area within the hibernaculum suitable for fungus growth [11, 17]. Alternatively, this intervention can be viewed as a physical removal of sediment ideal for $P$. destructans growth from the hibernaculum environment [17]. We modelled fungicide intervention by decreasing the $P$. destructans carrying capacity $K_{P d}$ by a proportion $\alpha$.

\subsubsection{Microclimate intervention}

Since $P$. destructans grows optimally in temperatures ranging from $12-15$ ${ }^{\circ} \mathrm{C}$, lowering temperatures below $12{ }^{\circ} \mathrm{C}$ could decrease the growth rate of $P$. destructans, potentially slowing or stopping the progression of infection once a bat becomes infected with WNS $[17,24]$. We modelled microclimate intervention by reducing the rate of WNS-induced mortality $\delta$ by a proportion $\alpha$. Note that since the proportion of non-moribund bats at the end of hibernation is determined by the expression $\frac{1}{s \delta+1}$, decreasing disease-induced mortality also increases the number of viable infected bats after hibernation.

\subsubsection{Soil bacteria intervention}

Several studies demonstrated the anti- $P$. destructans effects of volatile organic compounds produced by several species of soil bacteria $[32,33]$. As such, soil bacteria intervention is a contact-independent control that would inhibit conidial growth both within the environmental reservoir and on bat 
tissue. Therefore, we implement this control by scaling two parameters. We decrease both the natural growth rate of environmental $P$. destructans $\eta$ and the rate of bat progression from the exposed class to the infected class $\tau$ by a proportion $\alpha$.

\subsubsection{Ultraviolet (UV) light intervention}

$P$. destructans is extremely sensitive to UV light since it lacks a critical enzyme necessary for repair of cells damaged by UV radiation [34]. We hypothesise that bringing UV light sources to hibernacula would kill some fungus upon contact with light and also reduce growth of surviving fungus on bats and in the environmental reservoir. Hence, we model UV light intervention with three model modifications. We introduce a $P$. destructans mortality term $-\alpha P$ into each $\frac{\mathrm{d} P}{\mathrm{~d} t}$ equation to account for the loss of fungus on hibernaculum surfaces due to UV exposure. To account for the decrease in survival of fungal colonies on exposed bats, we reduce the rate of bat progression from the exposed class to the infected class $\tau$ by a proportion $\alpha$. Finally, we decrease the WNS-induced mortality rate $\delta$ by the same proportion $\alpha$ to account for non-lethal damage to fungus living on infected bats.

\subsubsection{Vaccination}

No vaccine for WNS exists, but a vaccine in the form of a topical gel or paste that could be sprayed on bats at roosting sites is currently in development [35, 36]. We incorporate vaccination with the addition of a vaccinated class $(V)$ to each subpopulation. After the end of the birth subphase but before resuming the regular roosting phase, a proportion $\alpha$ of susceptible bats are vaccinated and move to the vaccinated class, where they remain for the remainder of the simulation and are no longer susceptible to $P$. destructans infection. Note that $V$ class bats migrate during the dispersal reclassification in the same manner as all other bats, and are included in $N$ for reproduction purposes. Appendix A displays the modified model equations with vaccination.

\subsection{Parametrisation}

Table 1 summarises all of the parameters used in the model. Without the presence of disease $(\beta=\phi=0)$ a stable disease-free equilibrium exists, but due to natural bat mortality between the annual birth subphases the 
population carrying capacity $K_{M l}$ must actually be higher than the initial population size. For $K_{M l}$, we choose the smallest positive integer that would cause the population to reach 15,000 after every annual birth subphase (after transiently exceeding 15, 000 for the first few years of the simulation) in the absence of disease. Typically, 95\% of females each give birth to one pup over a 21-day period [19], resulting in a fecundity rate of $\gamma=0.5(0.95 / 21)$. In [17] it was estimated that the post-hibernation recovery probability of a viable infected bat is 0.75 , so in our model it corresponds to a rate of $a=0.75 / 92$. Banding studies have found that approximately $4 \%$ of little brown bats relocate between hibernacula and $12 \%$ relocate between summer roosting sites, for an overall $6 \%$ relocation rate any given year [20]. Therefore, we consider dispersal percentages ranging from 0 to 10 as biologically reasonable. In general, North American bat species return to the same hibernaculum with high fidelity year after year, so there is no need to consider population dynamics resulting from higher levels of dispersal. With the exception of the relative contributions of bat-to-bat and environment-to-bat contact to disease transmission, the remaining parameters are known biological constants or were well-approximated in [17].

Little is known about the relative contributions of bat-to-bat and environmentto-bat contact to WNS disease transmission, even though previous studies have identified this ratio as an important determinant of disease dynamics and control efficacy [17]. Hence, we conduct our model analyses for three disease transmission cases: primarily environment-to-bat contact, equal contributions of bat-to-bat and environment-to-bat contact and primarily batto-bat contact. In order to select corresponding $\phi$ and $\beta$ values for each disease case, we use the estimation that $25 \%$ of the population survives two years after the initial introduction of $P$. destructans [5]. Figure 2 shows the contour of $\beta, \phi$ values corresponding to $25 \%$ survival after two years in a single hibernaculum without control. The range of $\phi$ satisfying this survival criterion is approximately $\left(0,6.92 \cdot 10^{-13}\right)$. We define primarily environmentto-bat disease transmission to be located at $90 \%$ of the $\phi$ range along the contour, equal contributions of bat-to-bat and environment-to-bat transmission to be at $50 \%$ of the $\phi$ range along the contour and primarily bat-to-bat disease transmission to be at $10 \%$ of the $\phi$ range along the contour. The three disease cases we tested are summarised in Table 2. 


\subsection{Implementation}

We used the ode45 function in MATLAB $^{\circledR}$ (MathWorks ${ }^{\top M}$, R2018a) to numerically integrate the equations for each phase. Each simulation begins at the start of the swarming phase with the introduction of one exposed bat into a population of 14,999 susceptible bats with no existing $P$. destructans in the hibernaculum environment. The initial conditions for every subsequent phase are taken to be the final population values of the preceding phase.

\section{Results}

\subsection{Single subpopulation baseline}

Figure 3 displays the results of simulations for each control strategy at all possible disease transmission cases and intervention intensities. Note that the efficacy of each strategy in improving survival of little brown bats depends on both of these parameters.

The effectiveness of all control strategies increases with higher values of intervention intensity $\alpha$. Each strategy differs, however, in the slope of this correlation. Microclimate intervention is only effective at very high control intensities, while vaccination achieves $50 \%$ population survival at $\alpha \approx 0.18$. As shown, the other strategies fall between these extremes. Interestingly, the population survival curves with respect to intervention intensity for all control strategies except vaccination are convex, while the curve for vaccination is concave. Vaccination is also the only strategy whose effectiveness increases with time. In nearly every case, implementing a control strategy at any intervention intensity will improve bat population survival relative to no intervention. The only exception is microclimate intervention given primarily bat-to-bat disease transmission, which can actually decrease survival.

No single trend exists for the relationship between control efficacy and the route of disease transmission, since the control strategies target multiple transmission pathways. Microclimate intervention, soil bacteria intervention and vaccination are always more effective at higher contributions of bat-tobat contact to disease transmission. Fungicide intervention and UV light intervention, on the other hand, exhibit more complicated dynamics. At low control intensities a higher contribution of bat-to-bat contact to disease transmission yields better population survival, but at high control intensities this behaviour reverses and a higher contribution of environment-to-bat 
contact now results in better survival. The critical point at which the switch occurs differs between the two controls, occurring at $\alpha \approx 0.8$ for fungicide and at $\alpha \approx 0.37$ for UV light. Additional simulations reveal that these critical points shift lower when the total number of years simulated is reduced. UV light intervention is further anomalous for having the greatest difference between disease transmission cases. It is also important to note that disease transmission acts in a roughly linear way. That is, the survival percentage given equal contributions to disease transmission nearly always falls between survival percentages given primarily environment-to-bat transmissive contact and primarily bat-to-bat transmissive contact, the only exceptions occurring near the critical switching points of fungicide and UV light interventions.

At a realistic mid-range intervention intensity of $\alpha=0.67$ and with primarily environment-to-bat transmission, the most effective strategy is UV light intervention, followed in order by vaccination, fungicide intervention, soil bacteria intervention and microclimate intervention. If we instead consider equal contributions of environment-to-bat and bat-to-bat contact to disease transmission, the ranking of control strategies changes to the following: vaccination, UV light, fungicide, soil bacteria and microclimate. For primarily bat-to-bat transmissive contact, the ranking of most effective controls is as follows: vaccination, UV light, soil bacteria, fungicide and microclimate. These rankings remain largely the same across intervention intensities and simulation timespans, with only occasional isolated switches between adjacent strategies due to these factors.

\subsection{Multiple subpopulation results}

Our results demonstrate that dispersal has a significant impact on the survival of bat populations affected by WNS and can change the ranking of control strategies when optimising for overall combined survival of the two subpopulations. Whether dispersal increases or decreases survival, as well as the severity of its effects, depends on the primary route of disease transmission, the combination of control strategies and the intervention intensity.

The presence of dispersal can alter control efficacy to the point of reversing pairwise rankings of preferred control strategies. Figure 4 compares final population survival after 10 years given no dispersal with final population survival given $7 \%$ annual dispersal. The two subpopulations are completely autonomous in the absence of dispersal, so the ranking of control strategies in the left plot remains as established in Section 3.1. When 7\% of bats migrate, 
we observe that certain combinations of control strategies become more effective while others become less effective, which in a number of cases changes the ranking of preferred strategies. For example, implementing microclimate intervention in subpopulation $A$ and UV light intervention in subpopulation $B$ is preferable to the combination of microclimate intervention in $A$ and vaccination in $B$ given the absence of dispersal. Yet in the presence of $7 \%$ dispersal the ranking reverses, making microclimate intervention in $A$ and vaccination in $B$ a better combination than microclimate intervention in $A$ and UV light in $B$. Such ranking reversals can also occur when one of the subpopulations is left untreated. For instance, if a manager faces the choice of vaccinating one subpopulation of bats while leaving another untreated or applying fungicide to one and soil bacteria to the other, our model points to the latter option given the absence of dispersal. Incorporating dispersal, however, makes vaccinating one subpopulation and leaving the other untreated the better choice.

Many other examples of pairwise ranking reversals abound in Figure 4, all of which involve combinations where at least one of the controls in at least one pair is UV light or vaccination. The pairwise rankings of control combinations not including either UV light or vaccination in either pair remain the same, even though many of those control combinations do still increase or decrease in efficacy with the addition of dispersal. This principle also holds for lower intervention intensities, but at very high intervention intensities of $\alpha>0.97$ all of the control strategies become approximately equal in effectiveness, resulting in many more pairwise ranking reversals when adding dispersal, even among control combinations without UV light and vaccination. Note that because of the drastic differences in efficacy between controls at realistic intervention intensities, it is nearly always better to manage the total population by focusing efforts on the stronger control in one subpopulation instead of applying both controls at mid-range intervention intensities.

Figure 5 summarises the impact of dispersal on each control strategy individually. As dispersal increases, the effectiveness of vaccination increases while the effectiveness of all other strategies generally decreases. UV light intervention at mid-range intervention intensities of $0.4 \gtrsim \alpha \gtrsim 0.6$ is the only exception, since after initially decreasing in efficacy at low values of dispersal it begins to slowly become more effective as dispersal rises beyond 1.5\%. Vaccination is notable for being the most strongly impacted by dispersal in every situation. Notice that when each control strategy is viewed individually, vaccination and UV light intervention are the only ones that 
switch their rankings due to dispersal, since the other strategies are too far apart in terms of efficacy. As demonstrated above, combining one strategy in subpopulation $A$ with another strategy in subpopulation $B$ yields more interesting behaviour. Figure 6 generalises these results for all possible control strategy combinations at each of the three disease cases.

No single trend exists for the dynamics resulting from combinations of different controls in the two subpopulations. Figure 6 illustrates this by summarising the percent changes in survival due to dispersal. Notice that dispersal does not affect systems with the same control strategy in both subpopulations, since in that case the two subpopulations are identical throughout the course of the simulation. In combinations that pair vaccination with another control, the strong positive impact of dispersal on vaccination nearly always masks the weaker effects of dispersal on the other control. For example, consider the combination of vaccination and microclimate intervention. Since dispersal strongly increases the efficacy of vaccination but only slightly decreases the efficacy of microclimate intervention, implementing vaccination in one subpopulation and microclimate intervention in the other will still be positively affected by increased dispersal. Combinations of vaccination with UV light intervention given primarily bat-to-bat contact or equal contributions to disease transmission pose the only exception, since UV light in these cases is even more strongly affected by dispersal than vaccination is.

The dynamics are more varied in combinations not involving vaccination. Sometimes dispersal impacts the combination more negatively than it impacts each control individually, as is the case with fungicide in subpopulation $A$ and soil bacteria in subpopulation $B$ with disease transmission driven primarily by bat-to-bat contact. In other situations dispersal impacts the combination less negatively than it impacts each control individually, as when considering microclimate intervention in subpopulation $A$ and UV light intervention in subpopulation $B$ with disease transmission driven primarily by environment-to-bat contact. The lack of a single dynamical trend for pairings of different controls between the two subpopulations suggests that the $M$. lucifugus - $P$. destructans system exhibits underlying complexity due to multiple routes of disease transmission.

Surprisingly, the negative effects of dispersal are strongest when disease transmission is driven primarily by environment-to-bat contact (see Figure 6 ). The greatest negative percent change in survival due to dispersal occurs when soil bacteria intervention is implemented in one subpopulation and the other subpopulation is left untreated, with disease primarily trans- 
mitted by environment-to-bat contact: in this case dispersal of $8 \%$ creates a $<-26 \%$ change in survival relative to no dispersal. When disease is primarily transmitted by bat-to-bat contact, however, the greatest negative change in survival is merely $\approx-15 \%$, which results from applying fungicide intervention to one subpopulation and UV light intervention to the other. The positive impact of dispersal on vaccination, however, is not affected as much by changes in the route of disease transmission. Regardless of the combination of control strategies or the primary mode of disease transmission, the absolute value percent change in survival due to dispersal is greater at $8 \%$ dispersal than at $4 \%$ dispersal; that is, increasing the amount of dispersal will always intensify its impact on the population. Additional simulations also reveal that the effects of dispersal become more prominent over time in all situations.

\section{Discussion}

WNS is one of the most pressing wildlife diseases of this century [3, 5], necessitating prompt development of control strategies to mitigate its spread and progression. While conducting field trials of proposed controls in the wild might not be prudent or cost-effective, mathematical models can instead be used to easily test said strategies without endangering at-risk fragile ecosystems. Although bat migration is known to be a key part of the chain of transmission in emerging infectious diseases [37], nothing is known about the spatial dynamics of WNS. We posed and analysed a deterministic model of WNS in little brown bats that incorporates five promising control strategies targeting several disease mechanisms. Most importantly, we simultaneously considered the dynamics arising from an interplay between disease transmission, control strategies and bat migration. Our results demonstrate that the most effective management decisions must take interpopulation movement into account, and highlight the importance of further quantifying bat migratory dynamics in conjunction with WNS disease transmission. We showed that in many cases a given combination of controls can be among the most effective or among the least effective options depending on the presence and magnitude of bat dispersal.

Our single-subpopulation baseline analysis confirms the results established in [17]. In accordance with their findings, our results likewise call attention to the importance of understanding the relative contributions of 
environment-to-bat and bat-to-bat transmission. With respect to specific control strategies, in our model fungicide intervention is mathematically analogous to their Control IV (decreasing the environmental reservoir size) and microclimate intervention is mathematically analogous to Control I (thermal refugia), while the remainder of our controls are new. Since [17] only considered each control at intervention intensities sufficiently high to yield $33 \%$ or better five-year population survival, the study did not detect the novel dynamics we found at low intervention intensities. At high intervention intensities, however, our results concord with theirs: fungicide and microclimate interventions are plausible for situations in which disease transmission is driven primarily by environment-to-bat contact, although the latter requires drastic intervention intensities.

Incorporating dispersal is especially important when considering controls whose effectiveness is comparable in a single-population setting, as was the case for vaccination and UV light intervention in our model. If a manager has the resources to choose, for example, between applying fungicide intervention to one subpopulation and either vaccinating the other or treating it with UV light intervention, a model that does not take dispersal into account would predict that the latter option (treating the second subpopulation with UV light intervention) will result in higher survival. Factoring in the effects of a $7 \%$ annual dispersal rate, however, clearly points to vaccination in the second subpopulation as the better choice, especially in the long term (Figure 4). Failing to account for dispersal would, in this case, effectuate a management decision that results in over $15 \%$ lower population survival-a huge number for bat colonies near their tipping point. Since many real-world control strategies are likely to have comparable strengths, our study underscores the need for effective management decisions to always consider bat dispersal.

Our simulations also revealed that the effects of dispersal on control efficacy depend on the primary route of disease transmission. Previous studies illuminated that successful implementation of controls is highly dependent on the relative role of each transmission route [17]. In our spatial model those results are even more pronounced. The mode of disease transmission not only changes the effectiveness of individual controls, but also determines how dispersal affects each control. For example, while fungicide and microclimate interventions are much less effective in the presence of dispersal if disease is primarily transmitted by environment-to-bat contact, their efficacy remains virtually the same regardless of the dispersal amount if disease is instead transmitted primarily by bat-to-bat contact. This result suggests that em- 
pirical research investigating the migration dynamics of bats in conjunction with disease transmission is necessary to construct an accurate picture of WNS dynamics and, in turn, to develop more effective control strategies.

In general, our results indicate that most WNS control strategies should be expected to diminish in efficacy with higher levels of dispersal. Of the controls we tested, vaccination was the only one to benefit from the presence of bat dispersal. This accords with our simplifying assumptions that vaccination grants lifetime immunity and that vaccinated bats migrate in exactly the same way as non-vaccinated bats, thereby lessening the force of infection in every subpopulation they migrate to. Unless such a control can be developed and efficiently administered, managers should expect long-distance movements in bat populations to suppress the effectiveness of all control strategies.

We structured our model to investigate the spatial dynamics of WNS given the current understanding of the disease. Necessarily, this entailed making modelling assumptions that we believe are representative of actual disease dynamics and consistent with previous studies. Due to the rapid rate of research on WNS and North American bat species, our decisions, results and conclusions might need to be revisited in future studies once new data becomes available and additional control methods are proposed. Moreover, further research is needed to elucidate the impact of environmental and demographic stochasticity, as well as more complex migration schemes. While our simulations point to some controls as more effective than others, we did not consider potential differences between difficulty of implementation for the various control strategies. The present lack of extensive laboratory and field trials for the controls we tested implies that our results should be interpreted as provisional; whilst we established a ranking of control strategies, pragmatic concerns over safety and feasibility could limit the options available to managers.

In summary, we have provided a modelling approach that can be used to examine spatial dynamics arising from multiple transmission routes of whitenose syndrome in bats. We assessed the success or failure of several control strategies and, more consequentially, we demonstrated that those results are highly dependent on the presence and magnitude of dispersal. Additionally, our results accentuate the importance of considering bat dispersal together with the possible routes of disease transmission, since the emergent dynamics influence the way dispersal acts on the effectiveness of each control strategy. 


\section{Acknowledgements}

The authors thank Shandelle M. Henson for feedback.

\section{$6 \quad$ Funding}

MMM, JJP and IP were supported by the National Science Foundation, grant DMS 1659037; JD was supported by Summer Internship Funding from the Leadership, Innovation, and Liberal Arts Center at Bryn Mawr College.

\section{A Appendix}

We model the vaccination control strategy with the addition of a vaccinated class $(V)$ to each subpopulation. The equations below include the vaccinated class.

\section{A.0.1 Swarming phase}

The swarming phase equations with vaccination are given by:

$$
\begin{aligned}
& \frac{\mathrm{d} S}{\mathrm{~d} t}=-\phi P S-\mu S \\
& \frac{\mathrm{d} E}{\mathrm{~d} t}=\phi P S-\mu E \\
& \frac{\mathrm{d} V}{\mathrm{~d} t}=-\mu V \\
& \frac{\mathrm{d} P}{\mathrm{~d} t}=\eta P\left(1-\frac{P}{K_{P d}}\right)
\end{aligned}
$$

\section{A.0.2 Dispersal reclassification}

The dispersal reclassification equation with vaccination is given by:

$$
\left(\begin{array}{cccc}
S_{A_{1}} & E_{A_{1}} & I_{A_{1}} & V_{A_{1}} \\
S_{B_{1}} & E_{B_{1}} & I_{B_{1}} & V_{B_{1}}
\end{array}\right)=\left(\begin{array}{cc}
1-\sigma & \sigma \\
\sigma & 1-\sigma
\end{array}\right)\left(\begin{array}{cccc}
S_{A_{0}} & E_{A_{0}} & I_{A_{0}} & V_{A_{0}} \\
S_{B_{0}} & E_{B_{0}} & I_{B_{0}} & V_{B_{0}}
\end{array}\right)
$$




\section{A.0.3 Hibernation phase}

The hibernation phase equations with vaccination are given by:

$$
\begin{aligned}
\frac{\mathrm{d} S}{\mathrm{~d} t} & =-(\beta I+\phi P) S-\mu S \\
\frac{\mathrm{d} E}{\mathrm{~d} t} & =(\beta I+\phi P) S-(\tau+\mu) E \\
\frac{\mathrm{d} I}{\mathrm{~d} t} & =\tau E-(\delta+\mu) I \\
\frac{\mathrm{d} V}{\mathrm{~d} t} & =-\mu V \\
\frac{\mathrm{d} P}{\mathrm{~d} t} & =(\omega I+\eta P)\left(1-\frac{P}{K_{P d}}\right)
\end{aligned}
$$

\section{A.0.4 Roosting reclassification}

The roosting reclassification equations with vaccination are given by:

$$
\begin{aligned}
S_{1} & =S_{0} \\
E_{1} & =E_{0}+\frac{1}{s \delta+1} I_{0} \\
I_{1} & =0 \\
V_{1} & =V_{0} \\
P_{1} & =P_{0}
\end{aligned}
$$

\section{A.0.5 Roosting phase}

The roosting phase equations with vaccination are given by:

$$
\begin{aligned}
& \frac{\mathrm{d} S}{\mathrm{~d} t}=a E-\mu S \\
& \frac{\mathrm{d} E}{\mathrm{~d} t}=-a E-\mu E \\
& \frac{\mathrm{d} V}{\mathrm{~d} t}=-\mu V \\
& \frac{\mathrm{d} P}{\mathrm{~d} t}=\eta P\left(1-\frac{P}{K_{P d}}\right)
\end{aligned}
$$




\section{A.0.6 Roosting with birth}

The birth subphase equations with vaccination are given by:

$$
\begin{aligned}
\frac{\mathrm{d} S}{\mathrm{~d} t} & =\gamma N_{V}\left(1-\frac{N_{V}}{K_{M l}}\right)+a E-\mu S \\
\frac{\mathrm{d} E}{\mathrm{~d} t} & =-a E-\mu E \\
\frac{\mathrm{d} V}{\mathrm{~d} t} & =-\mu V \\
\frac{\mathrm{d} P}{\mathrm{~d} t} & =\eta P\left(1-\frac{P}{K_{P d}}\right)
\end{aligned}
$$

where $N_{V}=S+E+V$.

\section{A.0.7 Vaccination reclassification}

The vaccination reclassification equations are given by:

$$
\begin{aligned}
S_{1} & =(1-\alpha) S_{0} \\
E_{1} & =E_{0} \\
I_{1} & =I_{0} \\
V_{1} & =V_{0}+\alpha S_{0} \\
P_{1} & =P_{0}
\end{aligned}
$$




\section{References}

[1] Jeffrey M. Lorch, Carol U. Meteyer, Melissa J. Behr, Justin G. Boyles, Paul M. Cryan, Alan C. Hicks, Anne E. Ballmann, Jeremy T. H. Coleman, David N. Redell, DeeAnn M. Reeder, and et al. Experimental infection of bats with geomyces destructans causes white-nose syndrome. Nature, 480(7377):376-378, Oct 2011. doi: 10.1038/nature10590. URL http://dx.doi.org/10.1038/nature10590.

[2] Andrew M. Minnis and Daniel L. Lindner. Phylogenetic evaluation of geomyces and allies reveals no close relatives of pseudogymnoascus destructans, comb. nov., in bat hibernacula of eastern north america. Fungal Biology, 117(9): 638-649, Sep 2013. doi: 10.1016/j.funbio.2013.07.001. URL http://dx.doi.org/10.1016/j.funbio.2013.07.001.

[3] Jeffrey M. Lorch, Jonathan M. Palmer, Daniel L. Lindner, Anne E. Ballmann, Kyle G. George, Kathryn Griffin, Susan Knowles, John R. Huckabee, Katherine H. Haman, Christopher D. Anderson, and et al. First detection of bat white-nose syndrome in western north america. mSphere, 1(4), Aug 2016. doi: 10.1128/msphere.00148-16. URL http://dx.doi.org/10.1128/mSphere.00148-16.

[4] W. F. Frick, J. F. Pollock, A. C. Hicks, K. E. Langwig, D. S. Reynolds, G. G. Turner, C. M. Butchkoski, and T. H. Kunz. An emerging disease causes regional population collapse of a common north american bat species. Science, 329(5992):679-682, Aug 2010. doi: 10.1126/science.1188594. URL http://dx.doi.org/10.1126/science.1188594.

[5] D. S. Blehert, A. C. Hicks, M. Behr, C. U. Meteyer, B. M. BerlowskiZier, E. L. Buckles, J. T. H. Coleman, S. R. Darling, A. Gargas, R. Niver, and et al. Bat white-nose syndrome: An emerging fungal pathogen? Science, 323(5911):227-227, Jan 2009. doi: 10.1126/science.1163874. URL http://dx.doi.org/10.1126/science.1163874.

[6] J. G. Boyles, P. M. Cryan, G. F. McCracken, and T. H. Kunz. Economic importance of bats in agriculture. Science, 332(6025):41-42, Mar 2011. doi: 10.1126/science.1201366. URL http://dx.doi.org/10.1126/science. 1201366. 
[7] Thomas H. Kunz, Elizabeth Braun de Torrez, Dana Bauer, Tatyana Lobova, and Theodore H. Fleming. Ecosystem services provided by bats. Annals of the New York Academy of Sciences, 1223 (1):1-38, Mar 2011. doi: 10.1111/j.1749-6632.2011.06004.x. URL http://dx.doi.org/10.1111/j.1749-6632.2011.06004.x.

[8] Wayne E. Thogmartin, Carol A. Sanders-Reed, Jennifer A. Szymanski, Patrick C. McKann, Lori Pruitt, R. Andrew King, Michael C. Runge, and Robin E. Russell. White-nose syndrome is likely to extirpate the endangered indiana bat over large parts of its range. Biological Conservation, 160:162-172, Apr 2013. doi: 10.1016/j.biocon.2013.01.010. URL http://dx.doi.org/10.1016/j.biocon.2013.01.010.

[9] Richard A. Erickson, Wayne E. Thogmartin, Robin E. Russell, Jay E. Diffendorfer, and Jennifer A. Szymanski. A stage-structured, spatially explicit migration model for myotis bats: Mortality location affects system dynamics. Letters in Biomathematics, 1(2): 157-172, Jan 2014. doi: 10.1080/23737867.2014.11414477. URL http://dx.doi .org/10.1080/23737867 . 2014.11414477.

[10] Suzanne M. O'Regan, Krisztian Magori, J. Tomlin Pulliam, Marcus A. Zokan, RajReni B. Kaul, Heather D. Barton, and John M. Drake. Multi-scale model of epidemic fade-out: Will local extirpation events inhibit the spread of white-nose syndrome? Ecological Applications, 25(3):621-633, Apr 2015. doi: 10.1890/14-0417.1. URL http://dx.doi.org/10.1890/14-0417.1.

[11] Hannah T. Reynolds, Tom Ingersoll, and Hazel A. Barton. Modeling the environmental growth of pseudogymnoascus destructans and its impact on the white-nose syndrome epidemic. Journal of Wildlife Diseases, 51(2):318-331, Apr 2015. doi: 10.7589/2014-06-157. URL http://dx.doi.org/10.7589/2014-06-157.

[12] Robin E. Russell, Wayne E. Thogmartin, Richard A. Erickson, Jennifer Szymanski, and Karl Tinsley. Estimating the short-term recovery potential of little brown bats in the eastern united states in the face of white-nose syndrome. Ecological Modelling, 314: 111-117, Oct 2015. doi: 10.1016/j.ecolmodel.2015.07.016. URL http://dx.doi.org/10.1016/j.ecolmodel.2015.07.016. 
[13] Richard A. Erickson, Wayne E. Thogmartin, Jay E. Diffendorfer, Robin E. Russell, and Jennifer A. Szymanski. Effects of wind energy generation and white-nose syndrome on the viability of the indiana bat. PeerJ, 4:e2830, Dec 2016. doi: 10.7717/peerj.2830. URL http://dx.doi.org/10.7717/peerj. 2830.

[14] Kate E. Langwig, Joseph R. Hoyt, Katy L. Parise, Winifred F. Frick, Jeffrey T. Foster, and A. Marm Kilpatrick. Resistance in persisting bat populations after white-nose syndrome invasion. Philosophical Transactions of the Royal Society B: Biological Sciences, 372(1712):20160044, Dec 2016. doi: 10.1098/rstb.2016.0044. URL http://dx.doi.org/10.1098/rstb. 2016.0044.

[15] Brooke Maslo, Oliver C. Stringham, Amanda J. Bevan, Amanda Brumbaugh, Chris Sanders, MacKenzie Hall, and Nina H. Fefferman. High annual survival in infected wildlife populations may veil a persistent extinction risk from disease. Ecosphere, 8(12):e02001, Dec 2017. doi: 10.1002/ecs2.2001. URL http://dx.doi.org/10.1002/ecs2. 2001.

[16] Thomas G. Hallam and Gary F. McCracken. Management of the panzootic white-nose syndrome through culling of bats. Conservation Biology, 25(1):189-194, Nov 2010. doi: 10.1111/j.1523-1739.2010.01603.x. URL http://dx.doi.org/10.1111/j.1523-1739.2010.01603.x.

[17] A.D. Meyer, D.F. Stevens, and J.C. Blackwood. Predicting bat colony survival under controls targeting multiple transmission routes of white-nose syndrome. Journal of Theoretical Biology, 409:60-69, Nov 2016. doi: 10.1016/j.jtbi.2016.08.033. URL http://dx.doi.org/10.1016/j.jtbi.2016.08.033.

[18] Wayne H. Davis and Harold B. Hitchcock. Biology and migration of the bat, myotis lucifugus, in new england. Journal of Mammalogy, 46(2):296, May 1965. doi: 10.2307/1377850. URL http://dx.doi.org/10.2307/1377850.

[19] M. Brock Fenton and Robert M. R. Barclay. Myotis lucifugus. Mammalian Species, pages 1-8, Nov 1980. doi: 10.2307/3503792. URL http://dx.doi.org/10.2307/3503792. 
[20] Kaleigh J. O. Norquay, Felix Martinez-Nuñez, Jack E. Dubois, Kim M. Monson, and Craig K. R. Willis. Long-distance movements of little brown bats (myotis lucifugus). Journal of Mammalogy, 94(2):506-515, Apr 2013. doi: 10.1644/12-mamm-a-065.1. URL http://dx.doi.org/10.1644/12-mamm-a-065.1.

[21] Joseph R. Hoyt, Kate E. Langwig, J. Paul White, Heather M. Kaarakka, Jennifer A. Redell, Allen Kurta, John E. DePue, William H. Scullon, Katy L. Parise, Jeffrey T. Foster, and et al. Cryptic connections illuminate pathogen transmission within community networks. Nature, 563(7733):710-713, Nov 2018. doi: 10.1038/s41586-018-0720-z. URL http://dx.doi.org/10.1038/s41586-018-0720-z.

[22] Kate E. Langwig, Winifred F. Frick, Jason T. Bried, Alan C. Hicks, Thomas H. Kunz, and A. Marm Kilpatrick. Sociality, densitydependence and microclimates determine the persistence of populations suffering from a novel fungal disease, white-nose syndrome. Ecology Letters, 15(9):1050-1057, Jul 2012. doi: 10.1111/j.1461-0248.2012.01829.x. URL http://dx.doi.org/10.1111/j.1461-0248.2012.01829.x.

[23] K. E. Langwig, W. F. Frick, R. Reynolds, K. L. Parise, K. P. Drees, J. R. Hoyt, T. L. Cheng, T. H. Kunz, J. T. Foster, and A. M. Kilpatrick. Host and pathogen ecology drive the seasonal dynamics of a fungal disease, white-nose syndrome. Proceedings of the Royal Society B: Biological Sciences, 282(1799): 20142335-20142335, Dec 2014. doi: 10.1098/rspb.2014.2335. URL http://dx.doi.org/10.1098/rspb. 2014.2335.

[24] Justin G Boyles and Craig KR Willis. Could localized warm areas inside cold caves reduce mortality of hibernating bats affected by white-nose syndrome? Frontiers in Ecology and the Environment, 8(2):92-98, Mar 2010. doi: 10.1890/080187. URL http://dx.doi.org/10.1890/080187.

[25] Hjalmar R. Bouma, Hannah V. Carey, and Frans G. M. Kroese. Hibernation: the immune system at rest? Journal of Leukocyte Biology, 88(4):619-624, Jun 2010. doi: 10.1189/jlb.0310174. URL http://dx.doi.org/10.1189/jlb.0310174. 
[26] Michelle L. Verant, Justin G. Boyles, William Waldrep, Gudrun Wibbelt, and David S. Blehert. Temperature-dependent growth of geomyces destructans, the fungus that causes bat white-nose syndrome. PLoS ONE, 7(9):e46280, Sep 2012. doi: 10.1371/journal.pone.0046280. URL http://dx.doi.org/10.1371/journal.pone.0046280.

[27] Jeffrey M. Lorch, Laura K. Muller, Robin E. Russell, Michael O'Connor, Daniel L. Lindner, and David S. Blehert. Distribution and environmental persistence of the causative agent of whitenose syndrome, geomyces destructans, in bat hibernacula of the eastern united states. Applied and Environmental Microbiology, 79(4):1293-1301, Dec 2012. doi: 10.1128/aem.02939-12. URL http://dx.doi.org/10.1128/aem.02939-12.

[28] Paul M Cryan, Carol Meteyer, Justin G Boyles, and David S Blehert. Wing pathology of white-nose syndrome in bats suggests life-threatening disruption of physiology. BMC Biology, 8(1):135, 2010. doi: 10.1186/1741-7007-8-135. URL http://dx.doi.org/10.1186/1741-7007-8-135.

[29] L. Warnecke, J. M. Turner, T. K. Bollinger, V. Misra, P. M. Cryan, D. S. Blehert, G. Wibbelt, and C. K. R. Willis. Pathophysiology of white-nose syndrome in bats: a mechanistic model linking wing damage to mortality. Biology Letters, 9(4): 20130177-20130177, May 2013. doi: 10.1098/rsbl.2013.0177. URL http://dx.doi.org/10.1098/rsbl.2013.0177.

[30] Carol Uphoff Meteyer, Mick Valent, Jackie Kashmer, Elizabeth L. Buckles, Jeffrey M. Lorch, David S. Blehert, Amanda Lollar, Douglas Berndt, Emily Wheeler, C. LeAnn White, and et al. Recovery of little brown bats (myotis lucifugus) from natural infection with geomyces destructans, white-nose syndrome. Journal of Wildlife Diseases, 47(3):618-626, Jul 2011. doi: 10.7589/0090-3558-47.3.618. URL http://dx.doi.org/10.7589/0090-3558-47.3.618.

[31] Nathan W. Fuller, Jonathan D. Reichard, Morgan L. Nabhan, Spenser R. Fellows, Lesley C. Pepin, and Thomas H. Kunz. Freeranging little brown myotis (myotis lucifugus) heal from wing damage associated with white-nose syndrome. EcoHealth, 8(2): 
154-162, Jun 2011. doi: 10.1007/s10393-011-0705-y. URL http://dx.doi.org/10.1007/s10393-011-0705-y.

[32] Christopher T. Cornelison, Kyle T. Gabriel, Courtney Barlament, and Sidney A. Crow. Inhibition of pseudogymnoascus destructans growth from conidia and mycelial extension by bacterially produced volatile organic compounds. Mycopathologia, 177 (1-2):1-10, Nov 2013. doi: 10.1007/s11046-013-9716-2. URL http://dx.doi.org/10.1007/s11046-013-9716-2.

[33] Christopher T Cornelison, M Kevin Keel, Kyle T Gabriel, Courtney K Barlament, Trudy A Tucker, George E Pierce, and Sidney A Crow. A preliminary report on the contact-independent antagonism of pseudogymnoascus destructans by rhodococcus rhodochrousstrain dap96253. BMC Microbiology, 14(1), Sep 2014. doi: 10.1186/s12866-014-0246-y. URL http://dx.doi .org/10.1186/s12866-014-0246-y.

[34] Jonathan M. Palmer, Kevin P. Drees, Jeffrey T. Foster, and Daniel L. Lindner. Extreme sensitivity to ultraviolet light in the fungal pathogen causing white-nose syndrome of bats. Nature Communications, 9(1), Jan 2018. doi: 10.1038/s41467-017-02441-z. URL http://dx.doi.org/10.1038/s41467-017-02441-z.

[35] Tonie Rocke. The search for a vaccine to prevent whitenose syndrome. Echolocator, 6(1), Jan 2017. URL http://wiatri.net/Inventory/Bats/News/2017JanEcholocator.pdf.

[36] Ben Stading, James A. Ellison, William C. Carson, Panayampalli Subbian Satheshkumar, Tonie E. Rocke, and Jorge E. Osorio. Protection of bats (eptesicus fuscus) against rabies following topical or oronasal exposure to a recombinant raccoon poxvirus vaccine. PLOS Neglected Tropical Diseases, 11(10): e0005958, Oct 2017. doi: 10.1371/journal.pntd.0005958. URL http://dx.doi.org/10.1371/journal.pntd.0005958.

[37] J. C. Blackwood, D. G. Streicker, S. Altizer, and P. Rohani. Resolving the roles of immunity, pathogenesis, and immigration for rabies persistence in vampire bats. Proceedings of the National Academy of Sciences, 110(51):20837-20842, Dec 2013. doi: 10.1073/pnas.1308817110. URL http://dx.doi.org/10.1073/pnas.1308817110. 


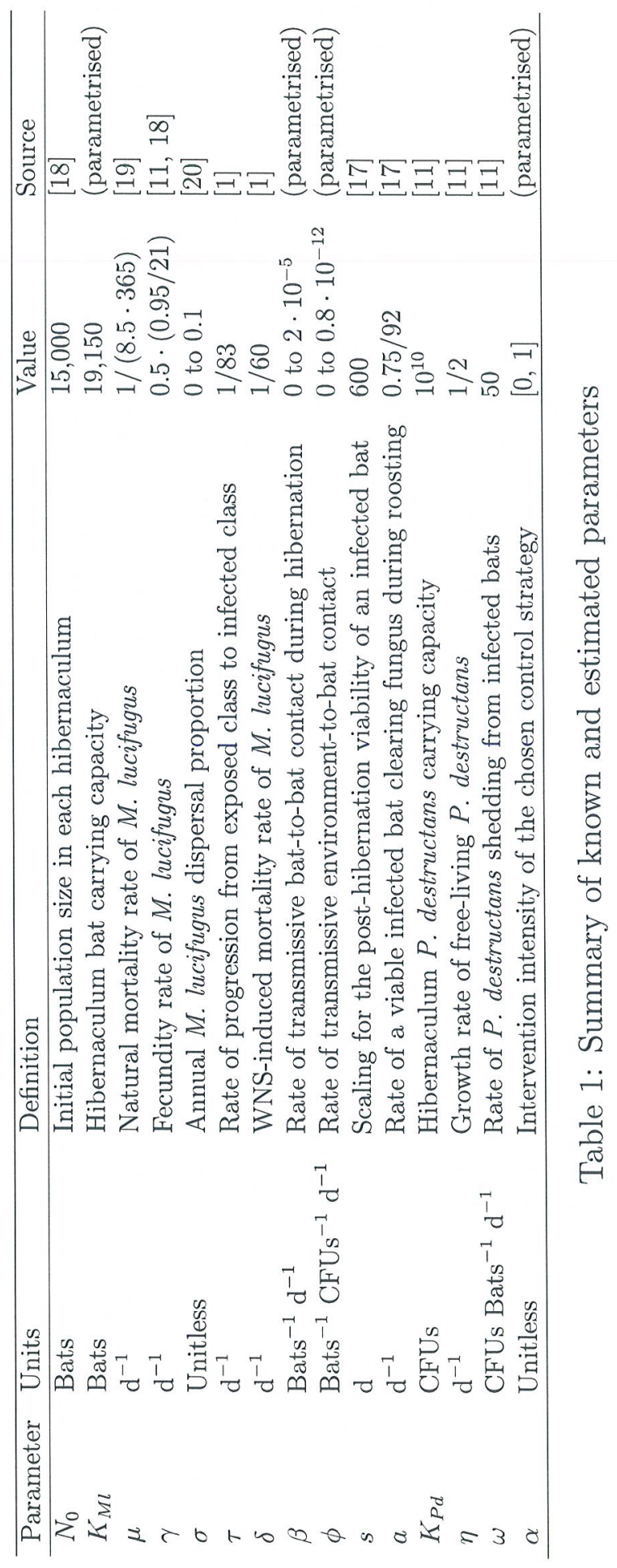




\begin{tabular}{lll}
\hline Disease Case & $\phi$ & $\beta$ \\
\hline Primarily environment-to-bat transmission & $6.23 \cdot 10^{-13}$ & $6.79 \cdot 10^{-7}$ \\
Equal contributions to transmission & $3.46 \cdot 10^{-13}$ & $3.89 \cdot 10^{-6}$ \\
Primarily bat-to-bat transmission & $6.92 \cdot 10^{-14}$ & $9.00 \cdot 10^{-6}$ \\
\hline
\end{tabular}

Table 2: Disease cases

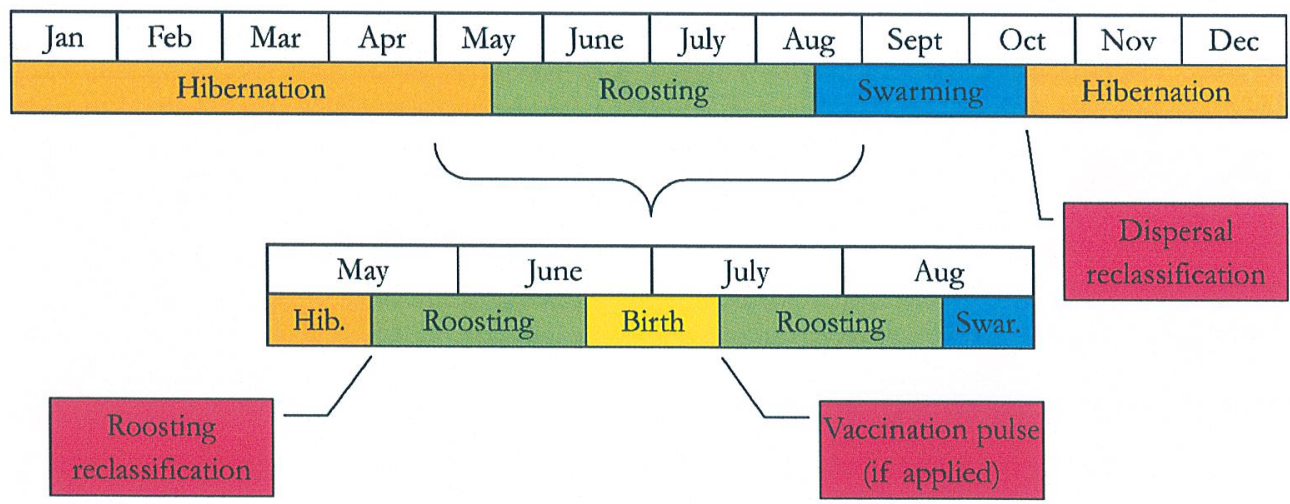

Figure 1: A schematic diagram illustrating the temporal arrangement of all model phases and discrete reclassification events. 


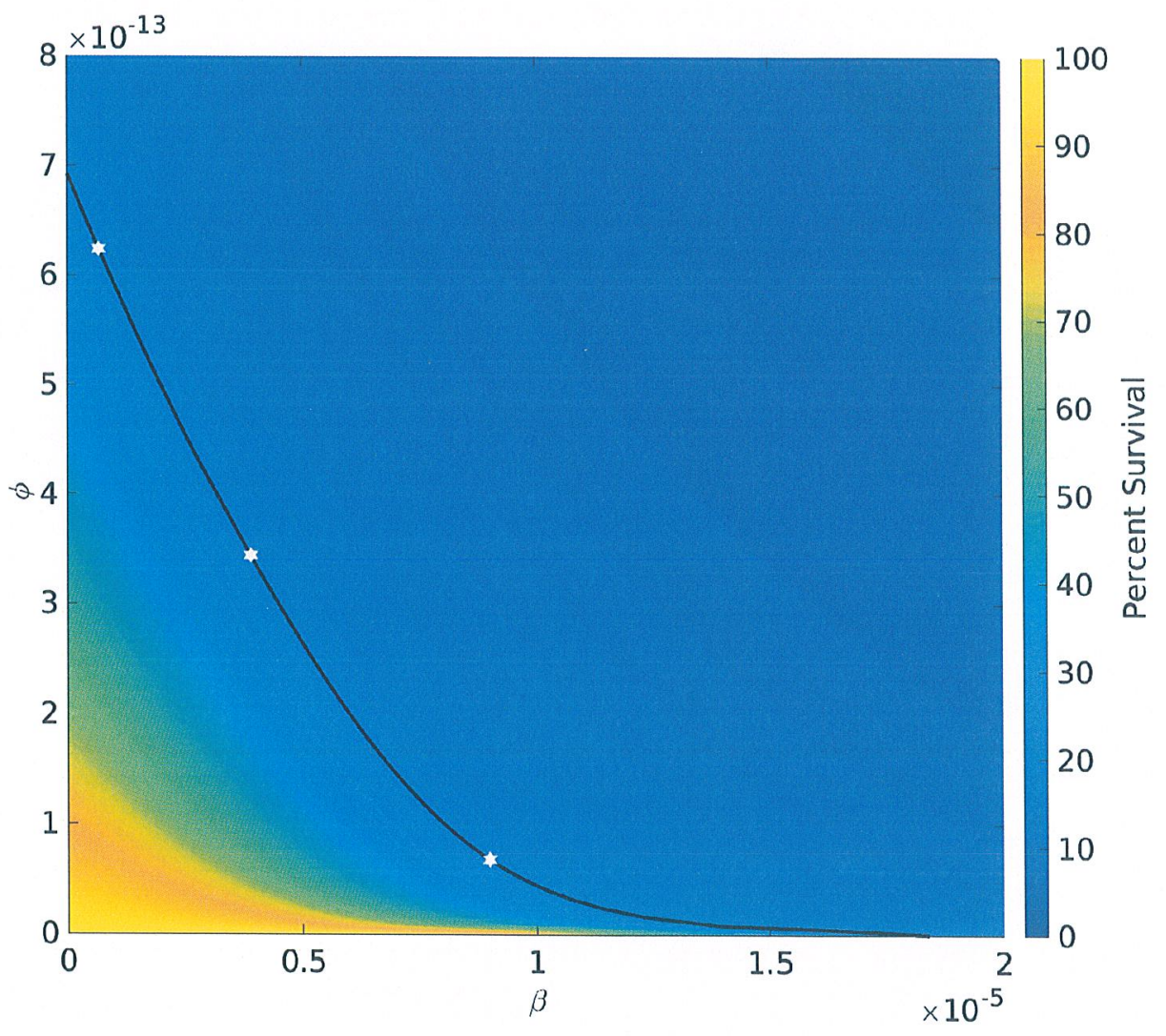

Figure 2: A heat map showing percent survival of a single population after two years. The black line indicates the $25 \%$ survival contour in $\beta, \phi$ space, with the three tested disease cases marked. 

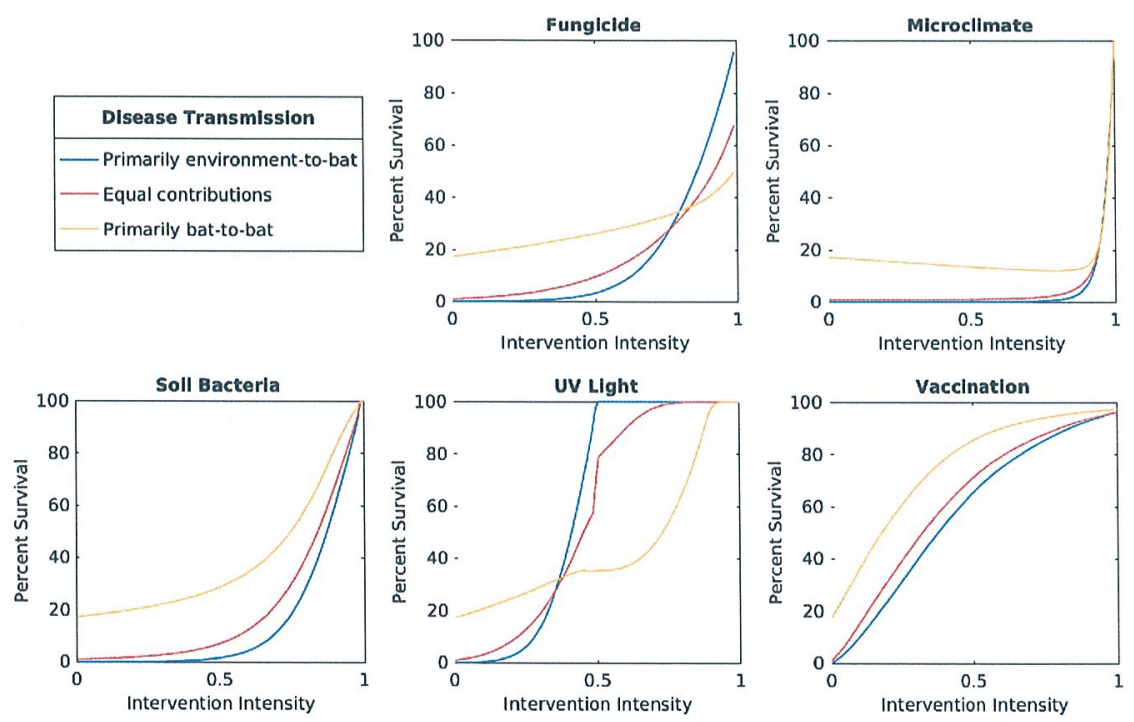

Figure 3: Each plot displays percent population survival against intervention intensity for all three disease transmission cases, with the plots differing only in the control strategy implemented. The population begins with 1 exposed bat and 14,999 susceptible bats and simulations are run for 10 years. 

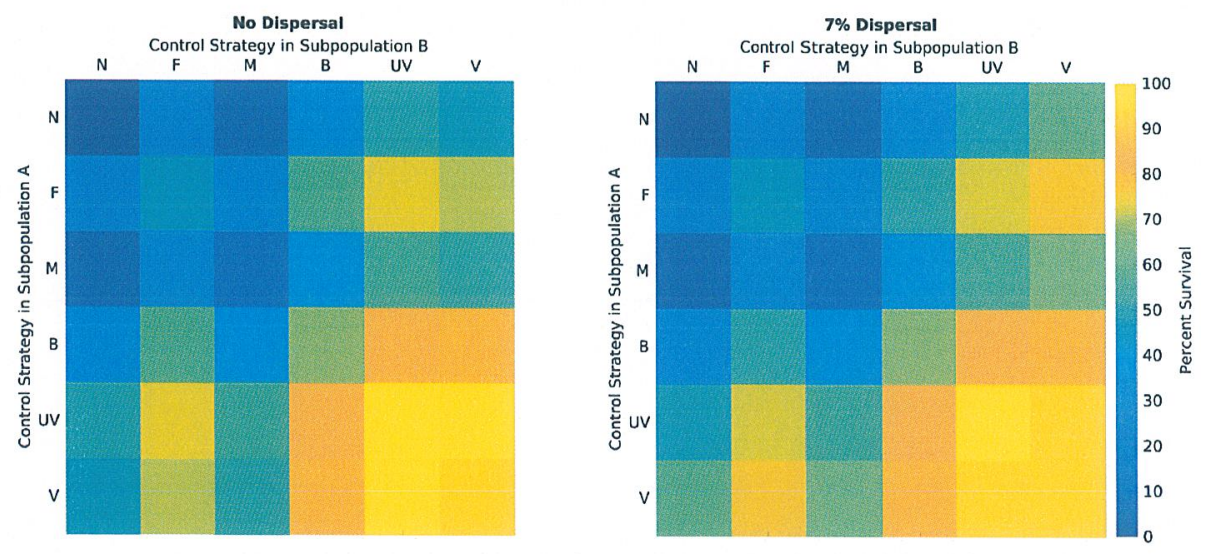

Figure 4: Each cell represents a separate simulation, with the colour denoting percent survival 10 years after a single exposed bat is introduced into a hibernaculum of 14,999 susceptible bats in each subpopulation. The letters denote controls as follows: $\mathrm{N}$ is no control, $\mathrm{F}$ is fungicide, $\mathrm{M}$ is microclimate, $\mathrm{B}$ is soil bacteria, UV is UV light and V is vaccination. The left plot has no dispersal, while the right plot has $7 \%$ annual dispersal. Disease is transmitted equally by environment-to-bat and bat-to-bat contact and the intervention intensity is $\alpha=0.9$. 

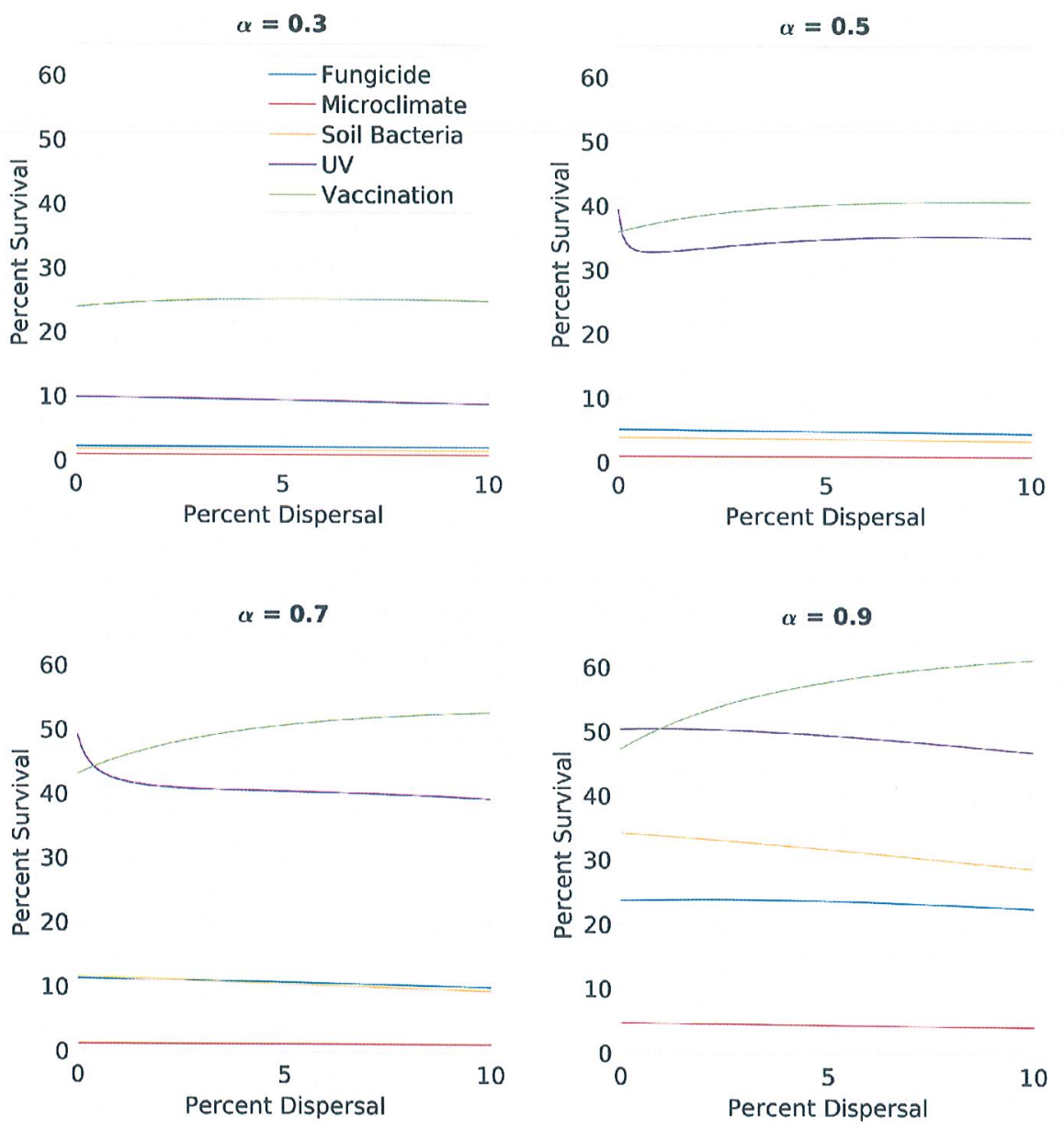

Figure 5: Each plot displays combined percent population survival for subpopulations A and B against dispersal amount for every control strategy, with the plots differing only in intervention intensity. Both subpopulations begin with 1 exposed bat and 14,999 susceptible bats, simulations are run for 10 years and equal contributions of environment-to-bat and bat-to-bat contact are assumed. 

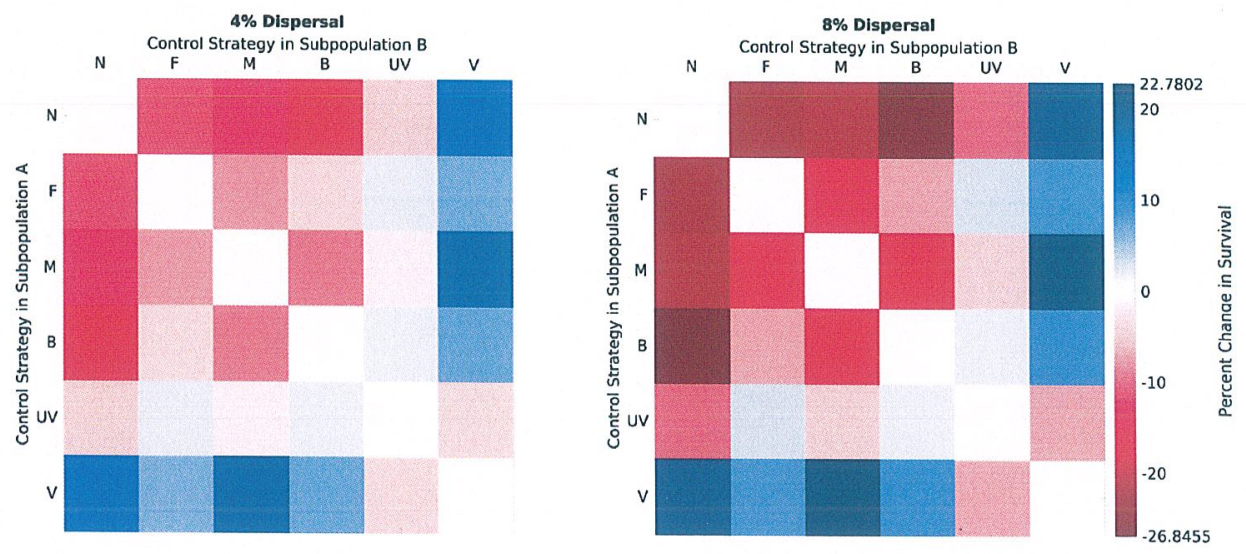

(a) Primarily environment-to-bat transmissive contact.
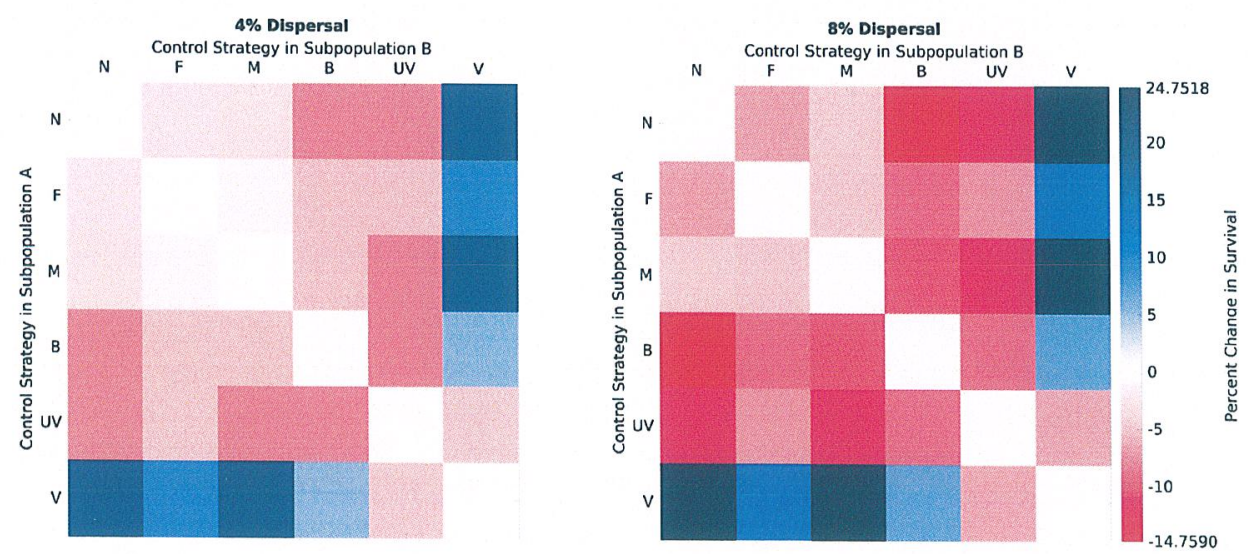

(b) Equal contributions of environment-to-bat and bat-to-bat contact to disease transmission. 

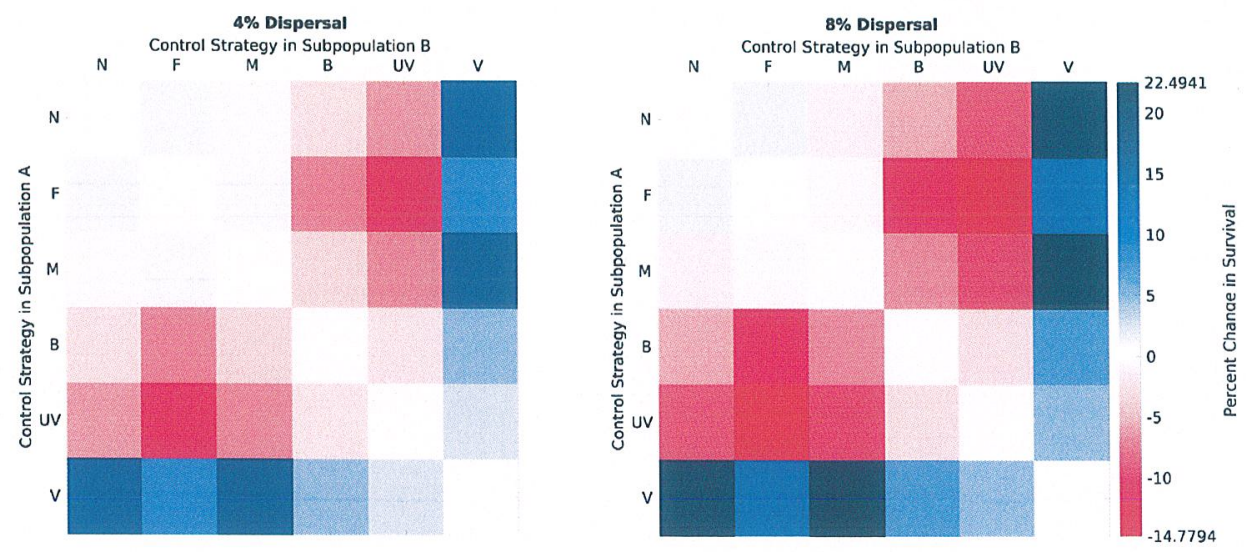

(c) Primarily bat-to-bat transmissive contact.

Figure 6: Each cell represents a separate simulation, with the colour denoting percent change in survival between no dispersal and annual dispersal values of $4 \%$ (left) or $8 \%$ (right). The letters denote controls as follows: $\mathrm{N}$ is no control, $\mathrm{F}$ is fungicide, $\mathrm{M}$ is microclimate, $\mathrm{B}$ is soil bacteria, UV is $\mathrm{UV}$ light and $\mathrm{V}$ is vaccination. The subfigures differ only in the mode of disease transmission. Both subpopulations begin with 1 exposed bat and 14,999 susceptible bats, simulations are run for 10 years and the intervention intensity is $\alpha=0.8$. 